\title{
Synchronization Control for A Class of Discrete-Time Dynamical Networks With Packet Dropouts: A Coding-Decoding-Based Approach
}

\author{
Licheng Wang, Zidong Wang, Fellow, IEEE, Qing-Long Han, Senior Member, IEEE, and Guoliang Wei
}

\begin{abstract}
The synchronization control problem is investigated for a class of discrete-time dynamical networks with packet dropouts via a coding-decoding-based approach. The data is transmitted through digital communication channels and only the sequence of finite coded signals is sent to the controller. A series of mutually independent Bernoulli distributed random variables is utilized to model the packet dropout phenomenon occurring in the transmissions of coded signals. The purpose of the addressed synchronization control problem is to design a suitable coding-decoding procedure for each node, based on which an efficient decoder-based control protocol is developed to guarantee that the closed-loop network achieves the desired synchronization performance. By applying a modified uniform quantization approach and the Kronecker product technique, criteria for ensuring the detectability of the dynamical network are established by means of the size of the coding alphabet, the coding period and the probability information of packet dropouts. Subsequently, by resorting to the input-to-state stability (ISS) theory, the desired controller parameter is obtained in terms of the solutions to a certain set of inequality constraints which can be solved effectively via available software packages. Finally, two simulation examples are provided to demonstrate the effectiveness of the obtained results.
\end{abstract}

Index Terms-Dynamical networks, coding-decoding, synchronization control, detectability analysis, input-to-state stability, packet dropouts.

\section{INTRODUCTION}

The past decade has seen significant progress in the field of complex networks because of their wide applications in a variety of practical systems such as World Wide Web, electric power grids, scientific citation web and biological networks [14], [24], [39], [44]. In general, a dynamical network typically consists of a number of interconnected dynamical nodes, in which each node can be viewed as a fundamental unit with

This work was supported in part by the Royal Society of the UK, the National Natural Science Foundation of China under Grants 61329301 and 61374039, the Australian Research Council Discovery Project under Grant DP160103567, the Hujiang Foundation of China under Grants C14002 and D15009, the Program for Capability Construction of Shanghai Provincial Universities under Grant 15550502500, and the Alexander von Humboldt Foundation of Germany. (Corresponding Author: Guoliang Wei.)

L. Wang and G. Wei are with the Shanghai Key Lab of Modern Optical System, Department of Control Science and Engineering, University of Shanghai for Science and Technology, Shanghai 200093, China. (Email: guoliang.wei1973@gmail.com)

Z. Wang is with the Department of Computer Science, Brunel University London, Uxbridge, Middlesex, UB8 3PH, United Kingdom. (Email: Zidong.Wang@brunel.ac.uk)

Q.-L. Han is with the School of Software and Electrical Engineering, Swinburne University of Technology, Melbourne, VIC 3122, Australia. (Email: qhaneswin.edu.au). its own physical meaning and the links exhibit particular network topology. In recent years, the dynamics analysis issues of complex networks have been drawing increasing research attention from communities including mathematics, physics, nonlinear science, and computer and information science, and a rich body of results has been available in the literature, see e.g. [2], [7], [23], [31], [36], [48].

The synchronization problem, which is concerned with a typical collective behavior in nature, has long been serving as an important research topic for dynamical networks because of its practical background and potential applications [4], [15], [17], [27], [42]. Roughly speaking, the synchronization phenomenon means that the state trajectories of all nodes tend to a certain identical value asymptotically. Recently, the synchronization problem for dynamical networks has attracted considerable research attention as evidenced by practical applications in a diverse range of areas such as the synchronization of sampling frequency in the industrial applications of wireless sensor networks [3], the phase synchronization of power networks [10], [43], the synchronization of memristor-based dynamical networks [13], and the global synchronization of public traffic road networks [1], to name just a few. However, in reality, there may be a case that the synchronization phenomenon cannot be attained for autonomous networks by their local connections, that is, the dynamic evolutions exhibit the asynchronous nature. For such kind of asynchronous networks, there is a vital need to develop effective synchronization control approaches in order to enforce the trajectories of all nodes to the desired synchronization manifold.

Concerning the synchronization control problems of dynamical networks, the available control strategies include, but are not limited to, the sampled-data control [37], the impulsive control [6], [16], [18], the pinning control [5], [30] and the intermittent control [20], [41]. Note that most reported synchronization control protocols have been assumed to work within the analog communication framework where the information is translated into electric pulses of varying amplitude. Compared with the traditional data transmission scheme in an analog manner, up to now, very little attention has been devoted to the synchronization control issue of dynamical networks with a digital communication mechanism, despite the fact that the digital information interaction has already become a dominant communication fashion for its obvious advantages of strong disturbance rejection capacity, low power consumption as well as high reliability. It is worth mentioning that, in the digitalization era, the digital 
communication strategy has been widely utilized in the areas of signal processing and control engineering.

In the real world, almost all the physical signals are virtually of analog nature and it would be improper to directly transmit them through digital channels. As such, before transmitting such analog signals in a digital pattern, one should first transform them into the digital form by an analog-to-digital (A/D) converter with the steps including sampling, reservation, quantization and encoding. Generally speaking, the sampling and reservation actions are mainly to convert a continuoustime signal into the discrete-time counterpart. The signal quantization aims to divide the continuous amplitudes of the analog signal into finite discrete values, while the signal encoding is intended to transform the quantized data into certain codewords by adopting some appropriate coding rules. To some extent, the data coding stage is most crucial in realizing the digitization transmission since it is closely associated with the detectability issue, i.e., whether and how the coded data could be recovered from the codewords with a prescribed accuracy requirement.

Compared with the traditional analog communication schemes, the distinct features of the digital communication protocols lie in that they a) have a good noise immunity; b) possess a high communication reliability; and c) are convenient for the data encryption. Here, the features a) and b) facilitate the long distant data transmission, and the feature c) prevents the transmitted data from being vulnerable to attacks launched by adversaries in response to the increasingly important security requirement of the information transmission. In particular, it is significantly important to design an appropriate coding-decoding procedure so as to ensure a satisfactory control performance via digital communication channels.

Presently, there have been primarily two methodologies in dealing with the decoder-based control protocols, namely, the information-theory-based method [11], [12] and the quantization-based method [34], [49]. With respect to the quantization-based method, the main idea is to design a suitable coding-decoding procedure to guarantee the convergence or boundedness of the error between the system state and the decoded state. In the past few years, the codingdecoding communication scheme has gained some initial research attention, see [22], [26], [35], [40] for some preliminary results. Up to now, almost all results concerning the codingdecoding issues have been on linear/nonlinear systems without network topology specifications, and the corresponding results on dynamical networks have not been addressed yet despite their wide applications in engineering practice.

The coding-decoding-based synchronization control problem for discrete-time dynamical networks appears to be an essentially difficult issue with three fundamental challenges identified as follows. 1) The first challenge stems from the mathematical difficulty in analyzing the addressed problem. As a typical dynamical network usually comes with both high dimension and tight coupling among the nodes, rather complicated/difficult mathematical analysis is expected and this is particularly true when the random packet dropout phenomenon is taken into account during the codeword transmissions [38]. On the other hand, due to the existence of the decoding error between the decoded system state and actual system state, it is fairly challenging to research into the issue of how to utilize imperfect decoded information to attain the desired control objective. In addition, since the performance of the coding-decoding protocol is influenced by a number of factors (e.g. the coding period, the size of the coding alphabet and the probabilistic packet dropouts), the third challenge is how to examine the effects of these combined factors on the prescribed system performance indices. It is, therefore, the main aim of this paper to deal with the listed challenges by addressing the synchronization control problem for dynamical networks using a coding-decoding-based approach.

Motivated by the above discussion, in this paper, we endeavor to launch a major study on the decoder-based synchronization control problem for a class of dynamical networks. A coding-decoding-based communication protocol is proposed to fit the digital data transmission with hope to enhance the communication quantity and information security. The main contributions of this paper are highlighted as follows: 1) the problem addressed is new in the sense that we make the first attempt to deal with the synchronization control problem for a class of dynamical networks via a coding-decodingbased method in the presence of probabilistic packet dropouts; 2) a modified uniform quantization approach is put forward so as to reduce possible conservatism in the design of the coding-decoding protocol; 3) the combined effects from the coding period, the size of coding alphabets and the random packet dropouts on the addressed problem are analyzed in a quantitative way; and 4) a set of easy-to-implement synchronization controllers is designed with explicitly characterized gain matrices by using the input-to-state stability (ISS) theory.

The rest of this paper is organized as follows. Section II briefly introduces the problems under consideration and presents the basic idea of the coding-decoding protocol. In Section III, both the coding-decoding-based detectability analysis and synchronization control problems are discussed for the dynamical network concerned. Two simulation examples are provided in Section IV to demonstrate the main results obtained and the paper is finally concluded in Section V.

Notation. The notations are quite standard. Throughout this paper, $\mathbb{R}^{n}$ and $\mathbb{R}^{n \times m}$ denote, respectively, the $n$-dimensional Euclidean space and the set of all $n \times m$ real matrices. $A^{T}$ represents the transpose of $A$ and $\operatorname{diag}_{N}\left\{A_{i}\right\}$ stands for the block-diagonal matrix $\operatorname{diag}\left\{A_{1}, A_{2}, \cdots, A_{N}\right\} . \operatorname{vec}_{N}\left\{x_{i}\right\}$ denotes $\left[\begin{array}{llll}x_{1}^{T} & x_{2}^{T} & \cdots & x_{N}^{T}\end{array}\right]^{T} . I_{n}$ is an $n$-dimensional identity matrix and $\mathbb{1}_{n}$ denotes an $n$ dimensional column vector with all one. $\lambda_{\max }\{A\} \quad\left(\lambda_{\min }\{A\}\right)$ is the maximum (minimum) eigenvalue of a symmetric matrix $A$. $|a|$ refers to the absolute value of a scalar $a$ and $\|x\|_{2}$ describes the Euclidean norm of a vector $x . \mathbb{E}\{x\}$ stands for the expectation of stochastic variable $x$. The symbol $\otimes$ denotes the Kronecker product. In symmetric block matrices, the asterisk $*$ is used to denote term that is induced by symmetry. 


\section{Problem Formulation And Preliminaries}

Consider a discrete-time dynamical network consisting of $N$ coupled nodes described by:

$$
\left\{\begin{aligned}
x_{i}(k+1) & =f\left(x_{i}(k)\right)+\sum_{j=1}^{N} w_{i j} \Gamma x_{j}(k)+u_{i}(k), \\
y_{i}(k) & =C_{i} x_{i}(k), \\
x_{i}(0) & =x_{i 0} \in \mathcal{X}_{0}, \quad i=1,2, \ldots, N
\end{aligned}\right.
$$

where, for the $i$ th node, $x_{i}(k) \in \mathbb{R}^{n}$ is the state vector which is inaccessible directly, and $y_{i}(k) \in \mathbb{R}^{m}$ and $u_{i}(k) \in \mathbb{R}^{n}$ are the measurement output and the control input, respectively. $\Gamma=\operatorname{diag}\left\{\gamma_{1}, \gamma_{2}, \ldots, \gamma_{n}\right\}$ is the inner coupling matrix linking the $j$ th state variable if $\gamma_{j} \neq 0 . W=\left[w_{i j}\right]_{N \times N}$ is the coupled configuration matrix of the network with $w_{i j} \geq 0(i \neq j)$ but not all zero. $x_{i 0}$ is the initial value of the $i$ th node belonging to a known set $\mathcal{X}_{0} . C_{i}$ is a constant matrix of appropriate dimensions. The nonlinear vector-valued function $f(\cdot): \mathbb{R}^{n} \mapsto$ $\mathbb{R}^{n}$ is continuous which satisfies $f(0)=0$ and the following condition:

$$
\left[f(x)-f(y)-U_{1}(x-y)\right]^{T}\left[f(x)-f(y)-U_{2}(x-y)\right] \leq 0
$$

for all $x, y \in \mathbb{R}^{n}$, where $U_{1}$ and $U_{2}$ are known real matrices of appropriate dimensions.

Note that full state information of the dynamical network (1) may not be obtained directly and only the network output is available. In this case, we construct the following state estimator for node $i$ :

$$
\left\{\begin{aligned}
\tilde{x}_{i}(k+1)= & f\left(\tilde{x}_{i}(k)\right)+\sum_{j=1}^{N} w_{i j} \Gamma \tilde{x}_{j}(k) \\
& +L_{i}\left(y_{i}(k)-C_{i} \tilde{x}_{i}(k)\right)+u_{i}(k), \\
\tilde{x}_{i}(0)= & \tilde{x}_{i 0} \in \mathcal{X}_{0}
\end{aligned}\right.
$$

where $\tilde{x}_{i}(k) \in \mathbb{R}^{n}$ is the estimator state for the $i$ th node, $L_{i} \in \mathbb{R}^{n \times m}$ is the estimator gain matrix to be designed and $\tilde{x}_{i 0}$ is the $i$ th estimator's initial condition.

Let $s(k) \in \mathbb{R}^{n}$ be the solution to the unforced isolate node

$$
\left\{\begin{aligned}
s(k+1) & =f(s(k)), \\
s(0) & =s_{0} \in \mathcal{X}_{0}
\end{aligned}\right.
$$

where $s_{0}$ is the initial condition.

Denote by $e_{s i}(k) \triangleq x_{i}(k)-s(k)$ the synchronization error vector. Then, for node $i$, we have the following synchronization error dynamics:

$$
\left\{\begin{aligned}
e_{s i}(k+1) & =f\left(e_{s i}(k)\right)+\sum_{j=1}^{N} w_{i j} \Gamma e_{s j}(k)+u_{i}(k), \\
e_{s i}(0) & =e_{s i 0} \in \mathcal{X}_{0}
\end{aligned}\right.
$$

where $f\left(e_{s i}(k)\right) \triangleq f\left(x_{i}(k)\right)-f(s(k))$ and $e_{s i 0} \triangleq x_{i 0}-s_{0}$ is the initial value.

To comply with the digital communication fashion, the purpose of this paper is to address the synchronization control problem for the dynamical network (1) by using a codingdecoding communication protocol. To be exact, by developing an efficient coding-decoding procedure based on the estimated network states, we shall design a decoder-based control protocol such that all the network nodes can be synchronized to the dynamics (4).

Before proceeding, a general form of the coding-decoding procedure for dynamical networks (1) is given as follows:

\section{Coder for Node $i$ :}

$$
g^{i}(l h)=\mathcal{F}_{l}^{i}\left(\tilde{x}_{i}(h), \tilde{x}_{i}(2 h), \ldots, \tilde{x}_{i}(l h)\right),
$$

\section{Decoder for Node $i$ :}

$$
\hat{X}^{i}(l h)=\mathcal{G}_{l}^{i}\left(\alpha_{i}(h) g^{i}(h), \alpha_{i}(2 h) g^{i}(2 h), \ldots, \alpha_{i}(l h) g^{i}(l h)\right)
$$

for $l=1,2, \ldots$, where $g^{i}(l h)$ is the codeword generated from the coder $i$ at the coding instant $l h$, and $\hat{X}^{i}(l h)$ is defined as

$$
\hat{X}^{i}(l h) \triangleq\left\{\hat{x}_{i}(l h), \hat{x}_{i}(l h+1), \ldots, \hat{x}_{i}((l+1) h-1)\right\}
$$

with $\hat{x}_{i}(k)$ being the decoded state of $\tilde{x}_{i}(k)$ for $k \in[l h,(l+$ 1)h). $\mathcal{F}_{l}^{i}(\cdot)$ and $\mathcal{G}_{l}^{i}(\cdot)$ are coder and decoder functions to be designed, respectively. $\alpha_{i}(l h)$ is a Bernoulli distributed random variable which characterizes the packet dropouts and satisfies the following probability distribution:

$$
\operatorname{Prob}\left\{\alpha_{i}(l h)=0\right\}=\bar{\alpha}_{i}, \quad \operatorname{Prob}\left\{\alpha_{i}(l h)=1\right\}=1-\bar{\alpha}_{i}
$$

where $\bar{\alpha}_{i} \in[0,1]$ is a given scalar. In addition, $\alpha_{i}(l h)$ and $\alpha_{j}(l h)$ are mutually independent random variables for $i \neq j$ with $i, j=1,2, \ldots, N$.

For node $i$, in terms of the decoded state $\hat{x}_{i}(k)$, the decoderbased synchronization controller is given as

$$
u_{i}(k)=K_{c}\left(\hat{x}_{i}(k)-s(k)\right)
$$

where $K_{c}$ is the controller parameter to be designed.

Remark 1: In the coding-decoding procedure (6)-(7), by employing the Bernoulli distributed white sequence $\alpha_{i}(l h)$, the phenomenon of probabilistic packet loss occurring in the transmission of codewords is well described, thereby better reflecting the reality. Also, because the coding period is $h$ times of the system sampling period, it can be observed from (7) that, for a fixed coding period $[l h,(l+1) h)$, the decoding function $\mathcal{G}_{l}^{i}(\cdot)$ of node $i$ applies the codeword $g^{i}(l h)$ to generate a set of decoded state $\hat{x}_{i}(k)(k \in[l h,(l+1) h))$ which indicates that the decoder can generate the decoded states at both the coding instant $l h$ and those non-coding time instants $l h+1, \cdots,(l+1) h-1$. Here, the decoded state $\hat{x}_{i}(k)$ $k \in(l h,(l+1) h)$ generated at non-coding instants can be viewed as a "prediction" of the actual system state.

For notation simplicity, we denote

$$
\begin{aligned}
x(k) & =\operatorname{vec}_{N}\left\{x_{i}(k)\right\}, \tilde{x}(k)=\operatorname{vec}_{N}\left\{\tilde{x}_{i}(k)\right\}, \\
\hat{x}(k) & =\operatorname{vec}_{N}\left\{\hat{x}_{i}(k)\right\}, e_{s}(k)=\operatorname{vec}_{N}\left\{e_{s i}(k)\right\}, \\
x_{0} & =\operatorname{vec}_{N}\left\{x_{i 0}\right\}, \tilde{x}_{0}=\operatorname{vec}_{N}\left\{\tilde{x}_{i 0}\right\}, \\
e_{s 0} & =\operatorname{vec}_{N}\left\{e_{s i 0}\right\}, F(x(k))=\operatorname{vec}_{N}\left\{f\left(x_{i}(k)\right\},\right. \\
F(\tilde{x}(k)) & =\operatorname{vec}_{N}\left\{f\left(\tilde{x}_{i}(k)\right\}, C=\operatorname{diag}_{N}\left\{C_{i}\right\},\right. \\
F\left(e_{s}(k)\right) & =\operatorname{vec}_{N}\left\{f\left(e_{s i}(k)\right)\right\}, L=\operatorname{diag}_{N}\left\{L_{i}\right\} .
\end{aligned}
$$

By recurring to the Kronecker product, the original dynamical network (1) can be rewritten in the following compact 
form:

$$
\left\{\begin{aligned}
x(k+1) & =F(x(k))+(W \otimes \Gamma) x(k)+u(k), \\
y(k) & =C x(k), \\
x(0) & =x_{0} .
\end{aligned}\right.
$$

Accordingly, we develop the following state estimator for the dynamical network (10):

$$
\left\{\begin{aligned}
\tilde{x}(k+1)= & F(\tilde{x}(k))+(W \otimes \Gamma) \tilde{x}(k) \\
& +L C(x(k)-\tilde{x}(k))+u(k), \\
\tilde{x}(0)= & \tilde{x}_{0}
\end{aligned}\right.
$$

and obtain the compact form of synchronization error dynamics (5) as follows:

$$
\left\{\begin{aligned}
e_{s}(k+1) & =F\left(e_{s}(k)\right)+(W \otimes \Gamma) e_{s}(k)+u(k), \\
e_{s}(0) & =e_{s 0} .
\end{aligned}\right.
$$

To facilitate the subsequent developments, we introduce the following definitions which are needed for stating the problem to be investigated.

Definition 1: Consider the discrete-time dynamical network of the compact form (10) subject to packet dropouts during the codeword transmissions. The network (10) is said to be detectable if there exist families of coder-decoder pairs (6) and (7) with a coding alphabet $\mathcal{H}$ of size $\chi$ such that

$$
\lim _{k \rightarrow \infty} \mathbb{E}\left\{\|x(k)-\hat{x}(k)\|_{2}\right\}=0
$$

holds for any solution of (10).

Definition 2: The discrete-time dynamical network (10) is said to be synchronized to the isolated node (4) if there exist families of decoder-controller pairs (8) such that

$$
\lim _{k \rightarrow \infty} \mathbb{E}\left\{\left\|e_{s}(k)\right\|_{2}\right\}=0
$$

holds for any solution of closed-loop system (12).

In this paper, we investigate the synchronization control problem for the dynamical network (10) by using a decoderbased control protocol. For the sake of realizing the synchronization criterion, one has to examine the detectability of the whole dynamical networks first. In other words, we are interested in developing an efficient coding-decoding procedure (6) and (7) for each node such that

a) The dynamical network (10) subject to the packet dropouts is detectable.

b) Based on the established detectability criterion, the dynamical network (10) is synchronized to the prescribed dynamics $s(k)$ by the decoder-based control protocol (8).

\section{Main Results}

In this section, our attention is focused on the analysis and design problems of the coding-decoding procedure for dynamical network (10). Then, based on the established codingdecoding procedure, we plan to deal with the decoder-based synchronization control problem for the addressed dynamical network to achieve the desired performance requirements.
To start with, we give the following useful lemma which will be needed for the subsequent derivation of our main results.

Lemma 1: Let the scalar $\mu_{1}>0$ be given. If there exist a positive definite matrix $P>0$ and a scalar $\varepsilon_{1}>0$ satisfying the following linear matrix inequality (LMI)

$$
\Pi_{1}=\left[\begin{array}{ccc}
-\Pi_{11} & -\varepsilon_{1} \Phi_{2 \Lambda}^{T} & (W \otimes \Gamma)^{T} P \\
* & -\varepsilon_{1} I_{N n} & P \\
* & * & -P
\end{array}\right]<0,
$$

then we have

$$
\left\|v_{1}(k+1)-v_{2}(k+1)\right\|_{2} \leq c_{0}\left\|v_{1}(k)-v_{2}(k)\right\|_{2}
$$

where $\Pi_{11}=\left(1+\mu_{1}\right) P-\varepsilon_{1} \Phi_{1 \Lambda}, \quad c_{0}=\sqrt{\beta}, \beta=$ $\frac{\left(1+\mu_{1}\right) \lambda_{\max }\{P\}}{\lambda_{\min }\{P\}}$, and $v_{1}(k)$ and $v_{2}(k)$ are any two solutions of $(10)$.

Proof: First, denoting $z(k) \triangleq v_{1}(k)-v_{2}(k)$ and $F(z(k)) \triangleq F\left(v_{1}(k)\right)-F\left(v_{2}(k)\right)$, it can be inferred immediately from $(10)$ that $z(k+1)=F(z(k))+(W \otimes \Gamma) z(k)$. Then, consider the Lyapunov function $V(k)=z^{T}(k) P z(k)$. The term $\Delta V(k)-\mu_{1} V(k)$, where $\Delta V(k)$ is the difference of $V(k)$ along the dynamics of $z(k+1)=F(z(k))+(W \otimes \Gamma) z(k)$, can be subsequently calculated as

$$
\begin{aligned}
& \Delta V(k)-\mu_{1} V(k) \\
= & z^{T}(k)\left[(W \otimes \Gamma)^{T} P(W \otimes \Gamma)-\left(1+\mu_{1}\right) P\right] z(k) \\
& +2 F^{T}(z(k)) P(W \otimes \Gamma) z(k)+F^{T}(z(k)) P F(z(k)) .
\end{aligned}
$$

It is easy to see from (2) that

$$
\left[\begin{array}{c}
z(k) \\
F(z(k))
\end{array}\right]^{T}\left[\begin{array}{cc}
\Phi_{1 \Lambda} & \Phi_{2 \Lambda}^{T} \\
* & I_{N n}
\end{array}\right]\left[\begin{array}{c}
z(k) \\
F(z(k))
\end{array}\right] \leq 0
$$

where $\Phi_{1 \Lambda}=I_{N} \otimes \Phi_{1}, \Phi_{2 \Lambda}=I_{N} \otimes \Phi_{2}, \Phi_{1}=\frac{U_{1}^{T} U_{2}+U_{2}^{T} U_{1}}{2}$ and $\Phi_{2}=-\frac{U_{1}+U_{2}}{2}$. Then, substituting (18) into (17) yields

$$
\begin{aligned}
& \Delta V(k)-\mu_{1} V(k) \\
\leq & z^{T}(k)\left[(W \otimes \Gamma)^{T} P(W \otimes \Gamma)-\left(1+\mu_{1}\right) P\right] z(k) \\
& +2 F^{T}(z(k)) P(W \otimes \Gamma) z(k)+F^{T}(z(k)) P F(z(k)) \\
& -\varepsilon_{1}\left[\begin{array}{c}
z(k) \\
F(z(k))
\end{array}\right]^{T}\left[\begin{array}{cc}
\Phi_{1 \Lambda} & \Phi_{2 \Lambda}^{T} \\
* & I_{N n}
\end{array}\right]\left[\begin{array}{c}
z(k) \\
F(z(k))
\end{array}\right] \\
= & \xi_{1}^{T}(k) \bar{\Pi}_{1} \xi_{1}(k)
\end{aligned}
$$

where

$$
\begin{aligned}
& \xi_{1}(k)=\left[\begin{array}{ll}
z^{T}(k) & F^{T}(z(k))
\end{array}\right]^{T}, \\
& \bar{\Pi}_{1}=\left[\begin{array}{cc}
\bar{\Pi}_{11} & (W \otimes \Gamma)^{T} P-\varepsilon_{1} \Phi_{2 \Lambda}^{T} \\
* & P-\varepsilon_{1} I_{N n}
\end{array}\right]
\end{aligned}
$$

with $\bar{\Pi}_{11}=(W \otimes \Gamma)^{T} P(W \otimes \Gamma)-\left(1+\mu_{1}\right) P-\varepsilon_{1} \Phi_{1 \Lambda}$.

By using the Schur Complement, it can be obtained from (15) that $\bar{\Pi}_{1}<0$, and therefore we have that $V(k+1) \leq$ $\left(1+\mu_{1}\right) V(k)$. In addition, it can be readily seen from the definition of $V(k)$ that $\lambda_{\min }\{P\}\|z(k+1)\|_{2}^{2} \leq V(k+1) \leq$ $\left(1+\mu_{1}\right) \lambda_{\max }\{P\}\|z(k)\|_{2}^{2}$, which implies that $\|z(k+1)\|_{2} \leq$ $\sqrt{\beta}\|z(k)\|_{2}$, namely, $\left\|v_{1}(k+1)-v_{2}(k+1)\right\|_{2} \leq c_{0} \| v_{1}(k)-$ $v_{2}(k) \|_{2}$. The proof is complete.

\section{B. State Estimator Design}


The following lemma provides a sufficient condition for the design problem of the state estimator (11) to ensure that the estimation error dynamics is convergent. Furthermore, the estimator gain matrix for each node is explicitly characterized.

Lemma 2: Let the positive scalar $0<\mu_{2}<1$ be given and consider the dynamical network (10) with estimator (11). It is assumed that there exist a positive definite matrix $Q=$ $\operatorname{diag}_{N}\left\{Q_{i}\right\}>0$, a matrix $X=\operatorname{diag}_{N}\left\{X_{i}\right\}$ and a positive scalar $\varepsilon_{2}>0$ satisfying

$$
\Pi_{2}=\left[\begin{array}{ccc}
-\left(1-\mu_{2}\right) Q-\varepsilon_{2} \Phi_{1 \Lambda} & -\varepsilon_{2} \Phi_{2 \Lambda}^{T} & \bar{Q} \\
* & -\varepsilon_{2} I_{N n} & Q \\
* & * & -Q
\end{array}\right]<0
$$

where $\bar{Q}=(Q(W \otimes \Gamma)-X C)^{T}$. Then, for the augmented dynamical network (10) and estimator (11), there always exist an integer $h \in \mathbb{Z}^{+}$and a scalar $c_{1}\left(0<c_{1}<1\right)$ such that

$$
\|x(k+h)-\tilde{x}(k+h)\|_{2}<c_{1}\|x(k)-\tilde{x}(k)\|_{2} .
$$

Moreover, the desired estimator gain matrix for each node can be obtained in the form of

$$
L_{i}=Q_{i}^{-1} X_{i}, \quad i=1,2, \ldots, N .
$$

Proof: Following the similar line in the proof of Lemma 1 , we denote the estimation error as $e(k) \triangleq x(k)-\tilde{x}(k)$. Then, subtracting (11) from (10) results in the estimation error dynamics

$$
e(k+1)=F(e(k))+(W \otimes \Gamma-L C) e(k),
$$

where $F(e(k)) \triangleq F(x(k))-F(\tilde{x}(k))$. Choosing the Lyapunov function as $V(k)=e^{T}(k) Q e(k)$, we have

$$
\begin{aligned}
& \Delta V(k)+\mu_{2} V(k) \\
= & {[F(e(k))+(W \otimes \Gamma-L C) e(k)]^{T} Q[F(e(k))} \\
& +(W \otimes \Gamma-L C) e(k)]-\left(1-\mu_{2}\right) e^{T}(k) Q e(k) \\
= & e^{T}(k)\left\{[(W \otimes \Gamma)-L C]^{T} Q[(W \otimes \Gamma)-L C]\right. \\
& \left.-\left(1-\mu_{2}\right) Q\right\} e(k)+2 F^{T}(e(k)) Q[(W \otimes \Gamma)-L C] e(k) \\
& +F^{T}(e(k)) Q F(e(k)) .
\end{aligned}
$$

Similarly, by noting (2), one has

$$
\left[\begin{array}{c}
e(k) \\
F(e(k))
\end{array}\right]^{T}\left[\begin{array}{cc}
\Phi_{1 \Lambda} & \Phi_{2 \Lambda}^{T} \\
* & I_{N n}
\end{array}\right]\left[\begin{array}{c}
e(k) \\
F(e(k))
\end{array}\right] \leq 0 .
$$

Then, it can be easily obtained from (23) and (24) that

$$
\begin{aligned}
& \Delta V(k)+\mu_{2} V(k) \\
\leq & e(k)^{T}\left[(W \otimes \Gamma-L C)^{T} Q(W \otimes \Gamma-L C)\right. \\
& \left.-\left(1-\mu_{2}\right) Q\right] e(k)+2 F^{T}(e(k)) Q(W \otimes \Gamma-L C) e(k) \\
& +F^{T}(e(k)) Q F(e(k)) \\
& -\varepsilon_{2}\left[\begin{array}{c}
e(k) \\
F(e(k))
\end{array}\right]^{T}\left[\begin{array}{cc}
\Phi_{1 \Lambda} & \Phi_{2 \Lambda}^{T} \\
* & I_{N n}
\end{array}\right]\left[\begin{array}{c}
e(k) \\
F(e(k))
\end{array}\right] \\
= & \xi_{2}^{T}(k) \bar{\Pi}_{2} \xi_{2}(k)
\end{aligned}
$$

where

$$
\begin{aligned}
\xi_{2}(k) & =\left[\begin{array}{ll}
e^{T}(k) & F^{T}(e(k))
\end{array}\right]^{T}, \\
\bar{\Pi}_{2} & =\left[\begin{array}{cc}
\bar{\Pi}_{21} & (W \otimes \Gamma-L C)^{T} Q-\varepsilon_{2} \Phi_{2 \Lambda}^{T} \\
* & Q-\varepsilon_{2} I_{N n}
\end{array}\right]
\end{aligned}
$$

with $\bar{\Pi}_{21}=(W \otimes \Gamma-L C)^{T} Q(W \otimes \Gamma-L C)-\left(1-\mu_{2}\right) Q-$ $\varepsilon_{2} \Phi_{1 \Lambda}$.

By noticing (22), it follows from (20) and the Schur Complement that $\Delta V(k)+\mu_{2} V(k) \leq 0$, which implies that $V(k+h) \leq\left(1-\mu_{2}\right) V(k+h-1) \leq\left(1-\mu_{2}\right)^{2} V(k+h-2) \leq$ $\cdots \leq\left(1-\mu_{2}\right)^{h} V(k)$. Based on such a fact, one can further obtain that

$$
\begin{aligned}
& \lambda_{\min }\{Q\}\|e(k+h)\|_{2}^{2} \leq V(k+h) \leq\left(1-\mu_{2}\right)^{h} V(k) \\
\leq & \left(1-\mu_{2}\right)^{h} \lambda_{\max }\{Q\}\|e(k)\|_{2}^{2},
\end{aligned}
$$

which means that

$$
\|x(k+h)-\tilde{x}(k+h)\|_{2}<c_{1}\|x(k)-\tilde{x}(k)\|_{2}
$$

where $c_{1}=\sqrt{\frac{\left(1-\mu_{2}\right)^{h} \lambda_{\max }\{Q\}}{\lambda_{\min }\{Q\}}}$. Therefore, it is not difficult to verify that there always exists a proper integer $h$ to ensure $0<c_{1}<1$. The proof of Lemma 2 is complete.

\section{Detectability Analysis}

Motivated by the uniform quantization approach proposed in [34], [49], in this paper, a modified uniform quantization approach is put forward, which would play an important role in the development of the coding-decoding procedure. For such a procedure, at each coding instant $l h$, since the current decoded state $\hat{x}_{i}(l h)$ of the $i$ th node is inaccessible at this stage, we introduce an auxiliary state $\bar{x}_{i}(l h)$ which is determined by the previously decoded state $\hat{x}_{i}(l h-1)$ and will be defined later, see (32). Then, a modified uniform quantization approach is applied to the error between the estimated state $\tilde{x}_{i}(l h)$ and the auxiliary state $\bar{x}_{i}(l h)$. In this regard, denote by $\delta_{i}(l h) \triangleq \tilde{x}_{i}(l h)-\bar{x}_{i}(l h)$ the error vector for the $i$ th node at the coding instant $l h$. In the following, a brief introduction of the proposed quantization method is given to facilitate the readers. For presentation convenience, sometimes, the arguments of a function or a matrix will be omitted in the analysis when no confusion can arise.

For the given scaling parameters $a_{i}>0(i=1,2, \ldots, N)$ and integer $q$, we can partition the hyperrectangles $\mathcal{B}_{a_{i}}=$ $\left\{\delta_{i} \in \mathbb{R}^{n}:\left|\delta_{i}^{(j)}\right| \leq a_{i}, j=1, \ldots, n\right\}$ into $q^{n}$ hyperrectangles $I_{s_{1}^{i}}^{i 1}\left(a_{i}\right) \times I_{s_{2}^{i}}^{i 2}\left(a_{i}\right) \times \cdots \times I_{s_{n}^{i}}^{i n}\left(a_{i}\right)$, where $s_{1}^{i}, s_{2}^{i}, \ldots, s_{n}^{i} \in$ $\{1,2, \ldots, q\}$ and

$$
\begin{aligned}
& I_{1}^{i j}\left(a_{i}\right) \triangleq\left\{\delta_{i}^{(j)} \mid-a_{i} \leq \delta_{i}^{(j)}<-a_{i}+\frac{2 a_{i}}{q}\right\}, \\
& I_{2}^{i j}\left(a_{i}\right) \triangleq\left\{\delta_{i}^{(j)} \mid-a_{i}+\frac{2 a_{i}}{q} \leq \delta_{i}^{(j)}<-a_{i}+\frac{4 a_{i}}{q}\right\}, \\
& \vdots \\
& I_{q}^{i j}\left(a_{i}\right) \triangleq\left\{\delta_{i}^{(j)} \mid a_{i}-\frac{2 a_{i}}{q} \leq \delta_{i}^{(j)} \leq a_{i}\right\}
\end{aligned}
$$

where $\delta_{i}^{(j)}$ is the $j$ th element of the vector $\delta_{i}$. As a result, for 
each $\mathcal{B}_{a_{i}}$, the center of the hyperrectangle $I_{s_{1}^{i}}^{i 1}\left(a_{i}\right) \times I_{s_{2}^{i}}^{i 2}\left(a_{i}\right) \times$ $\cdots \times I_{s_{n}^{i}}^{i n}\left(a_{i}\right)$ can be defined as

$$
\eta_{a_{i}}^{i}\left(s_{1}^{i}, s_{2}^{i}, \ldots, s_{n}^{i}\right) \triangleq\left[\begin{array}{llll}
b_{i 1} & b_{i 2} & \ldots & b_{i n}
\end{array}\right]^{T}
$$

where $b_{i j}=-a_{i}+\frac{\left(2 s_{j}^{i}-1\right) a_{i}}{q}, j=1,2, \ldots, n$.

Hence, for any $\delta_{i} \in \mathcal{B}_{a_{i}}$, there exist unique integers $s_{1}^{i}, s_{2}^{i}, \ldots, s_{n}^{i} \in\{1,2, \ldots, q\}$ such that $\delta_{i} \in I_{s_{1}^{i}}^{i 1}\left(a_{i}\right) \times$ $I_{s_{2}^{i}}^{i 2}\left(a_{i}\right) \times \cdots \times I_{s_{n}^{i}}^{i n}\left(a_{i}\right)$, which amounts to the following

$$
\left\|\delta_{i}-\eta_{a_{i}}^{i}\left(s_{1}^{i}, s_{2}^{i}, \ldots, s_{n}^{i}\right)\right\|_{2} \leq \frac{\sqrt{n} a_{i}}{q} .
$$

In the coding-decoding procedure to be proposed, each segment $I_{s_{j}^{i}}^{i j}\left(a_{i}\right)(j=1,2, \ldots, n)$ of the hyperrectangle $\mathcal{B}_{a_{i}}$ determines an integer $s_{j}^{i}$, which is one component of the codeword. So, the transmitted codeword corresponds to a certain integer sequence $s_{1}^{1}, \ldots, s_{n}^{1}, s_{1}^{2}, \ldots, s_{n}^{2}, \ldots, s_{1}^{N}, \ldots, s_{n}^{N}$, which is vitally important to the decoding procedure.

Assume that Lemma 1 and Lemma 2 hold for some proper constants $c_{0}$ and $c_{1}$, respectively. The following specific form of coding-decoding procedure is proposed to handle the detectability analysis problem for the $i$ th node.

Coder for Node $i$ : For $\delta_{i}(l h) \triangleq \tilde{x}_{i}(l h)-\bar{x}_{i}(l h) \in$ $I_{s_{1}^{i}}^{i 1}\left(a_{i}(l h)\right) \times I_{s_{2}^{i}}^{i 2}\left(a_{i}(l h)\right) \times \cdots \times I_{s_{n}^{i}}^{i n}\left(a_{i}(l h)\right) \subset \mathcal{B}_{a_{i}(l h)}$, we have

$$
g^{i}(l h)=\left[s_{1}^{i}, s_{2}^{i}, \ldots, s_{n}^{i}\right]
$$

where $\bar{x}_{i}(l h)$ is defined by

$$
\begin{aligned}
\bar{x}_{i}(0) & =0, \\
\bar{x}_{i}(k) & =\hat{x}_{i}(k), k \neq l h, \\
\bar{x}_{i}(l h) & =f\left(\hat{x}_{i}(l h-1)\right)+\sum_{j=1}^{N} w_{i j} \Gamma \hat{x}_{j}(l h-1)+u_{i}(l h-1) .
\end{aligned}
$$

\section{Decoder for Node $i$ :}

$$
\begin{aligned}
\hat{x}_{i}(0) & =0, \\
\hat{x}_{i}(k+1) & =f\left(\hat{x}_{i}(k)\right)+\sum_{j=1}^{N} w_{i j} \Gamma \hat{x}_{j}(k)+u_{i}(k), k \neq l h-1, \\
\hat{x}_{i}(l h) & =\bar{x}_{i}(l h)+\alpha_{i}(l h) \eta_{a_{i}(l h)}^{i}\left(s_{1}^{i}, s_{2}^{i}, \ldots, s_{n}^{i}\right) .
\end{aligned}
$$

By utilizing the Kronecker product, we reformulate the compact coding-decoding procedure as follows.

Coder : For $\delta(l h) \triangleq \tilde{x}(l h)-\bar{x}(l h) \in I_{s_{1}^{1}}^{11}\left(a_{1}(l h)\right) \times$ $\cdots \times I_{s_{n}^{1}}^{1 n}\left(a_{1}(l h)\right) \times I_{s_{1}^{2}}^{21}\left(a_{2}(l h)\right) \times \cdots \times I_{s_{n}^{2}}^{2 n}\left(a_{2}(l h)\right) \times \cdots \times$ $I_{s_{1}^{N}}^{N 1}\left(a_{N}(l h)\right) \times \cdots \times I_{s_{n}^{N}}^{N n}\left(a_{N}(l h)\right) \subset \mathcal{B}_{a(l h)}$, we have

$$
g(l h)=\left[s_{1}^{1}, \ldots, s_{n}^{1}, s_{1}^{2}, \ldots, s_{n}^{2}, \ldots, s_{1}^{N}, \ldots, s_{n}^{N}\right]
$$

where $\mathcal{B}_{a(l h)}=\left\{x(l h) \in \mathbb{R}^{N n}:\left|x^{(j)}(l h)\right|<a(l h), j=\right.$ $1, \ldots, N n\}$ and $\bar{x}(l h)=\operatorname{vec}_{N}\left\{\bar{x}_{i}(l h)\right\}$ is defined by

$$
\begin{aligned}
\bar{x}(0) & =0, \\
\bar{x}(k) & =\hat{x}(k), \quad k \neq l h, \\
\bar{x}(l h) & =F(\hat{x}(l h-1))+(W \otimes \Gamma) \hat{x}(l h-1)+u(l h-1) .
\end{aligned}
$$

\section{Decoder :}

$$
\begin{aligned}
\hat{x}(0) & =0, \\
\hat{x}(k+1) & =F(\hat{x}(k))+(W \otimes \Gamma) \hat{x}(k)+u(k), \quad k \neq l h-1, \\
\hat{x}(l h) & =\bar{x}(l h)+\alpha(l h) \eta_{a(l h)}\left(s_{1}^{1}, \ldots, s_{n}^{N}\right)
\end{aligned}
$$

where $a(l h)=\max _{1 \leq i \leq N}\left\{a_{i}(l h)\right\}, \quad \alpha(l h)=$ $\operatorname{diag}_{N}\left\{\alpha_{i}(l h) I_{n}\right\}$ and $\eta_{a(l h)}\left(s_{1}^{1}, \ldots, s_{n}^{N}\right)=\operatorname{vec}_{N}\left\{\eta_{a_{i}(l h)}^{i}\left(s_{1}^{i}\right.\right.$, $\left.\left.s_{2}^{i}, \ldots, s_{n}^{i}\right)\right\}$.

In the following, some definitions are given to facilitate the subsequent developments.

$$
\begin{aligned}
s_{0} & \triangleq \sup _{x_{i 0} \in \mathcal{X}_{0}}\left\|x_{i 0}\right\|_{2}^{2}, \\
a(h) & \triangleq\left(2 c_{1}+c_{0}^{h}\right) \sqrt{N s_{0}} \\
\mathbb{E}\{a((l+1) h)\} & \triangleq 2 c_{1}^{l} \sqrt{N s_{0}}\left(c_{1}+c_{0}^{h}\right)+\tilde{\alpha} \sqrt{N n} c_{0}^{h} a(l h)
\end{aligned}
$$

where $\tilde{\alpha}=\frac{\bar{\alpha}}{q}+(1-\bar{\alpha})$ and $\bar{\alpha}=\prod_{i=1}^{N}\left(1-\bar{\alpha}_{i}\right)$.

The following lemma shows that the coding-decoding protocol developed in this paper is well posed. That is, the decoding condition $\mathbb{E}\{\tilde{x}(l h)-\bar{x}(l h)\} \in \mathcal{B}_{a(l h)}$ holds for all $l=1,2, \ldots$

Lemma 3: The coding-decoding procedure (34)-(36) satisfies the following constraint

$$
\mathbb{E}\left\{\|\tilde{x}(l h)-\bar{x}(l h)\|_{\infty}\right\} \leq a(l h), l=1,2, \ldots .
$$

Proof: This lemma can be proved by using the mathematical induction. First, for $l=1$, by considering Lemma 1, Lemma 2 and the property of vector norm, we have

$$
\begin{aligned}
& \mathbb{E}\left\{\|\tilde{x}(h)-\bar{x}(h)\|_{2}\right\} \\
\leq & \mathbb{E}\left\{\|\tilde{x}(h)-x(h)\|_{2}\right\}+\mathbb{E}\left\{\|x(h)-\bar{x}(h)\|_{2}\right\} \\
\leq & c_{1}\|e(0)\|_{2}+c_{0}\|x(h-1)-\hat{x}(h-1)\|_{2} \\
\leq & 2 c_{1} \sqrt{N s_{0}}+c_{0}^{h}\|x(0)-\hat{x}(0)\|_{2},
\end{aligned}
$$

which guarantees

$$
\mathbb{E}\left\{\|\tilde{x}(l h)-\bar{x}(l h)\|_{\infty}\right\} \leq a(l h) .
$$

Subsequently, assuming that $\mathbb{E}\left\{\|\tilde{x}(j h)-\bar{x}(j h)\|_{\infty}\right\} \leq$ $\sqrt{N n} \mathbb{E}\{a(j h)\}$ for all $j=2, \ldots, l$, we arrive at

$$
\begin{aligned}
& \mathbb{E}\left\{\|\tilde{x}((l+1) h)-\bar{x}((l+1) h)\|_{2}\right\} \\
\leq & \mathbb{E}\left\{\|\tilde{x}((l+1) h)-x((l+1) h)\|_{2}\right\} \\
& +\mathbb{E}\left\{\|x((l+1) h)-\bar{x}((l+1) h)\|_{2}\right\} \\
\leq & c_{1}^{l+1}\|e(0)\|_{2}+c_{0} \mathbb{E}\left\{\|x(l h+h-1)-\hat{x}(l h+h-1)\|_{2}\right\} \\
\leq & 2 c_{1}^{l+1} \sqrt{N s_{0}}+c_{0}^{h} \mathbb{E}\left\{\|x(l h)-\hat{x}(l h)\|_{2}\right\} .
\end{aligned}
$$

Furthermore, taking the packet dropout phenomenon into account, we obtain

$$
\begin{aligned}
& \|x(l h)-\hat{x}(l h)\|_{2} \\
= & \left\|x(l h)-\bar{x}(l h)-\alpha(l h) \eta_{a(l h)}\left(s_{1}^{1}, \ldots, s_{n}^{N}\right)\right\|_{2} \\
\leq & \|x(l h)-\tilde{x}(l h)\|_{2}+\| \tilde{x}(l h)-\bar{x}(l h) \\
& \quad-\alpha(l h) \eta_{a(l h)}\left(s_{1}^{1}, \ldots, s_{n}^{N}\right) \|_{2} \\
\leq & 2 c_{1}^{l} \sqrt{N s_{0}}+\left\|\tilde{x}(l h)-\bar{x}(l h)-\alpha(l h) \eta_{a(l h)}\left(s_{1}^{1}, \ldots, s_{n}^{N}\right)\right\|_{2} .
\end{aligned}
$$

Since the codeword loss occurs in a random way for each node and is governed by a set of mutually independent 
Bernoulli distributed sequence $\alpha_{i}(l h)$, the term $\| \tilde{x}(l h)-$ $\bar{x}(l h)-\alpha(l h) \eta_{a(l h)}\left(s_{1}^{1}, \ldots, s_{n}^{N}\right) \|_{2}$ in (42) can be further rewritten as

$$
\begin{aligned}
& \left\|\tilde{x}(l h)-\bar{x}(l h)-\alpha(l h) \eta_{a(l h)}\left(s_{1}^{1}, \ldots, s_{n}^{N}\right)\right\|_{2} \\
= & \prod_{i=1}^{N} \alpha_{i}(l h)\left\|\tilde{x}(l h)-\bar{x}(l h)-\eta_{a(l h)}\left(s_{1}^{1}, \ldots, s_{n}^{N}\right)\right\|_{2} \\
& +\sum_{j=1}^{N} \prod_{i=1, i \neq j}^{N}\left(1-\alpha_{i}(l h)\right) \alpha_{j}(l h) \| \tilde{x}(l h)-\bar{x}(l h) \\
& -\operatorname{diag}\{\underbrace{I_{n}, \ldots, I_{n}}_{j-1}, 0, \underbrace{\underbrace{}_{n}, \ldots, I_{n}}_{N-j}\} \eta_{a(l h)}\left(s_{1}^{1}, \ldots, s_{n}^{N}\right) \|_{2} \\
& +\cdots+\prod_{i=1}^{N}\left(1-\alpha_{i}(l h)\right)\|\tilde{x}(l h)-\bar{x}(l h)\|_{2}
\end{aligned}
$$

where the first term on the right-hand side of (43) implies the case that there is no packet dropout occurring during the codeword transmissions, the second term describes that only one node is subject to the codeword loss and the last term indicates that all the nodes lose the codewords at the transmission stage.

For presentation convenience, only some cases of packet dropouts for the dynamical network are described in (43). Specifically, without loss of generality, we assume that the first $m$ nodes suffer from the packet dropouts where $m \in$ $\{1,2, \ldots, N\}$. Then, we have

$$
\begin{aligned}
& \| \tilde{x}(l h)-\bar{x}(l h)-\operatorname{diag}\{\underbrace{0, \ldots, 0}_{m}, \underbrace{I_{n}, \ldots, I_{n}}_{N-m}\} \\
& \times \eta_{a(l h)}\left(s_{1}^{1}, \ldots, s_{n}^{N}\right) \|_{2} \\
= & \| \tilde{x}(l h)-\bar{x}(l h)-\eta_{a(l h)}\left(s_{1}^{1}, \ldots, s_{n}^{N}\right) \\
& +\operatorname{diag}\{\underbrace{I_{n}, \ldots, I_{n}}_{m}, \underbrace{0, \ldots, 0}_{N-m}\} \eta_{a(l h)}\left(s_{1}^{1}, \ldots, s_{n}^{N}\right) \|_{2} \\
\leq & \left\|\tilde{x}(l h)-\bar{x}(l h)-\eta_{a(l h)}\left(s_{1}^{1}, \ldots, s_{n}^{N}\right)\right\|_{2} \\
& +\left\|[\underbrace{\eta_{a^{1}(l h)}^{1 T}, \ldots, \eta_{a^{m}(l h)}^{m T}}_{m}, \underbrace{0, \ldots, 0}_{N-m}]^{T}\right\|_{2} \\
\leq & \sqrt{N n} \frac{a(l h)}{q}+\sqrt{N n}\left(a(l h)-\frac{a(l h)}{q}\right) \\
= & \sqrt{N n} a(l h) .
\end{aligned}
$$

It is not difficult to verify that, if we consider the case of packet dropouts for arbitrary $m$ nodes, the above result still holds. Accordingly, it can be derived from (42)-(44) that

$$
\begin{aligned}
\mathbb{E}\left\{\|x(l h)-\hat{x}(l h)\|_{2}\right\} \leq & 2 c_{1}^{l} \sqrt{N s_{0}}+\sqrt{N n} \frac{\bar{\alpha}}{q} \mathbb{E}\{a(l h)\} \\
& +\sqrt{N n}(1-\bar{\alpha}) \mathbb{E}\{a(l h)\} .
\end{aligned}
$$

Thus, it can be concluded from (37), (41) and (45) that

$$
\mathbb{E}\left\{\|\tilde{x}((l+1) h)-\bar{x}((l+1) h)\|_{2}\right\} \leq a((l+1) h),
$$

which means

$$
\mathbb{E}\left\{\|\tilde{x}((l+1) h)-\bar{x}((l+1) h)\|_{\infty}\right\} \leq a((l+1) h) .
$$

Consequently, it follows that the Lemma 3 holds for all $l \geq 1$, and the proof of this lemma is complete.

The following theorem offers a sufficient condition to guarantee the detectability of the dynamical network (10) by utilizing the proposed coding-decoding protocol.

Theorem 1: The dynamical network (10) is detectable with the coding-decoding procedure (34)-(36) if the inequality

$$
\left(\frac{\bar{\alpha}}{q}+1-\bar{\alpha}\right) \sqrt{N n} c_{0}^{h}<1
$$

holds subject to (15) and (20) for some positive integer $q$, where $c_{0}$ and $h$ satisfy Lemma 1 and Lemma 2, respectively.

Proof: It follows from (37), (48) and $0<c_{1}<1$ that $\lim _{l \rightarrow+\infty} \mathbb{E}\{a(l h)\}=0$, which indicates

$$
\lim _{l \rightarrow \infty} \mathbb{E}\left\{\|\tilde{x}(l h)-\bar{x}(l h)\|_{2}\right\}=0 .
$$

Then, it can be easily seen from (45) that, at each coding instant $l h$, we have

$$
\lim _{l \rightarrow \infty} \mathbb{E}\left\{\|x(l h)-\hat{x}(l h)\|_{2}\right\}=0 .
$$

On the other hand, when $l h<k<(l+1) h$, noticing that $x(k)$ and $\hat{x}(k)$ can be viewed as two solutions of (10), one finds $\mathbb{E}\left\{\|x(k)-\hat{x}(k)\|_{2}\right\} \leq c_{0}^{k-l h} \mathbb{E}\left\{\|x(l h)-\hat{x}(l h)\|_{2}\right\}$ by Lemma 1 , which implies that $\mathbb{E}\left\{\|x(k)-\hat{x}(k)\|_{2}\right\}$ is also bounded at non-coding instants. Therefore, it follows immediately from the above analysis that

$$
\lim _{k \rightarrow \infty} \mathbb{E}\left\{\|x(k)-\hat{x}(k)\|_{2}\right\}=0,
$$

which ends the proof.

Remark 2: In Theorem 1, a criterion has been established to ensure the detectability of the dynamical network (10). It is not difficult to find that all the information (including the system matrices, the coding period, the size of coding alphabet and the statistics characteristics of packet dropouts) are reflected in the condition (48). Hence, the detectability of the dynamical network (10) depends heavily on the combined influences of those factors mentioned above. Let us now evaluate the impact on the performance of the proposed coding-decoding protocol from the following three aspects. 1) From (48), one has $q>\frac{\bar{\alpha} \sqrt{N n} c_{0}^{h}}{1-(1-\bar{\alpha}) \sqrt{N n} c_{0}^{h}}$, which requires that the size of the coding alphabet $\mathcal{H}$ should satisfy $\chi>\frac{\left(\bar{\alpha} \sqrt{N n} c_{0}^{h}\right)^{N n}}{\left(1-(1-\bar{\alpha}) \sqrt{N n} c_{0}^{h}\right)^{N n}}$. Especially, when $\bar{\alpha}=1$, namely, there is no packet dropout occurring, (48) reduces to $q>\sqrt{N n} c_{0}^{h}$, then the size $\chi$ is required to satisfy $\chi>\left(\sqrt{N n} c_{0}\right)^{N n h}$.2) The inequality (48) also indicates $1-\bar{\alpha}<\frac{q \sqrt{N n} c_{0}^{-h}-1}{q-1}$. Recalling the definition of $\bar{\alpha}$, it is noticed that the term $1-\bar{\alpha}$ can be regarded as the total packet dropout probability of the whole network. Therefore, it is easily seen that the upper bound of admissible data packet dropout probability is $\frac{q \sqrt{N n} c_{0}^{-h}-1}{q-1}$. 3) For a given size $\chi$ of the coding alphabet $\mathcal{H}$, with increased packet dropout probabilities $\bar{\alpha}_{i}$, the $\bar{\alpha}$ becomes smaller and thus inevitably weakens the feasibility of the inequality (48) and even leads to the possible divergence of the coding-decoding algorithm. 
Accordingly, this gives a tradeoff between the packet dropout probabilities and the convergence of the proposed algorithm.

\section{Synchronization Control}

Based on the established results of the detectability analysis problem in the last subsection, now let us tackle the decoderbased synchronization control problem for the dynamical network (10). Due to the existence of control input error induced by the decoding error, the ISS analysis approach is employed.

For the convenience of the readers, let us recall some basic notions and necessary foundations on the ISS theory before driving our main results.

Consider the following discrete-time nonlinear system

$$
x(k+1)=f(x(k), u(k))
$$

where $x(k) \in \mathbb{R}^{n}$ is the system state vector, $u(k) \in \mathbb{R}^{p}$ is the control input and $f(\cdot, \cdot): \mathbb{R}^{n} \times \mathbb{R}^{p} \mapsto \mathbb{R}^{n}$ is a continuous function satisfying $f(0,0)=0$.

First, let us introduce some important function classes which will be used in the subsequent developments. $\mathcal{K}, \mathcal{K}_{\infty}$ and $\mathcal{K} \mathcal{L}$ denote the different classes of functions. A function $\gamma(\cdot)$ : $\mathbb{R}^{+} \mapsto \mathbb{R}^{+}$belongs to class $\mathcal{K}$ if it is a continuous strictly increasing function with $\gamma(0)=0$, and belongs to class $\mathcal{K}_{\infty}$ if $\gamma(\cdot) \in \mathcal{K}$ with $\gamma(r) \mapsto \infty$ as $r \mapsto \infty$. Also, a function $\sigma(s, k): \mathbb{R}^{+} \times \mathbb{R}^{+} \mapsto \mathbb{R}^{+}$belongs to class $\mathcal{K} \mathcal{L}$ if, for each fixed $k$, the function $\sigma(\cdot, k) \in \mathcal{K}$, and for each fixed $s$, the function $\sigma(s, \cdot)$ is decreasing and $\sigma(\cdot, k) \mapsto 0$ as $k \mapsto \infty$. A function is said to be a $\mathcal{K}\left(\mathcal{K}_{\infty}\right.$ or $\left.\mathcal{K} \mathcal{L}\right)$ class function if it belongs to the class $\mathcal{K}\left(\mathcal{K}_{\infty}\right.$ or $\left.\mathcal{K} \mathcal{L}\right)$.

Definition 3: The system (52) is said to be input-to-state stable if there exist a $\mathcal{K} \mathcal{L}$ class function $\beta(\cdot, \cdot)$ and a $\mathcal{K}$ class function $\gamma(\cdot)$ such that the evolution of the system state $x(k)$ satisfies

$$
\|x(k)\|_{2} \leq \beta\left(\|x(0)\|_{2}, k\right)+\gamma\left(\|u(k)\|_{\infty}\right)
$$

for $\forall k \geq 0$ and $\forall x(0) \in \mathbb{R}^{n}$, where $\|u(k)\|_{\infty} \triangleq$ $\sup _{k}\left\{\|u(k)\|_{2}\right\}$.

Remark 3: It is clear from (53) that the bound of the state $x(k)$ is closely associated with the initial condition $x(0)$ and the system input $u(k)$. To be specific, if the system input $u(k)$ is bounded, then the state evolution trajectory $x(k)$ is naturally bounded. Also, it follows from the properties of functions $\beta(\cdot, \cdot)$ and $\gamma(\cdot)$ that the state $x(k)$ is ultimately bounded as $k$ increases. In addition, as pointed out in [21], the ISS property further implies the case of "converging-input" to "convergingstate", i.e., the system state $x(k)$ converges to 0 if the input $u(k)$ achieves 0 as $k \mapsto \infty$.

To proceed, we present the following lemma.

Lemma 4: [21] The nonlinear discrete-time system (52) is said to be input-to-state stable if there exist a positive definite function $V(k, x(k)):[0,+\infty) \times \mathbb{R}^{n} \mapsto \mathbb{R}$ (called an ISSLyapunov function), three $\mathcal{K}_{\infty}$ class functions $\alpha_{1}(\cdot), \alpha_{2}(\cdot)$ and $\alpha_{3}(\cdot)$, and a $\mathcal{K}$ class function $\varpi(\cdot)$ such that the following two inequalities

$$
\alpha_{1}\left(\|x(k)\|_{2}\right) \leq V(k, x(k)) \leq \alpha_{2}\left(\|x(k)\|_{2}\right),
$$

$$
\begin{aligned}
& V(k+1, x(k+1))-V(k, x(k)) \\
\leq & -\alpha_{3}\left(\|x(k)\|_{2}\right)+\varpi\left(\|u(k)\|_{2}\right)
\end{aligned}
$$

hold for all $x(k) \in \mathbb{R}^{n}$ and $u(k) \in \mathbb{R}^{p}$. Furthermore, if the above two inequalities are met simultaneously, then the functions $\beta(\cdot, \cdot)$ and $\gamma(\cdot)$ in Definition 3 can be selected as $\beta(\cdot, k)=\alpha_{1}^{-1}\left(\phi^{k} \alpha_{2}(\cdot)\right)(0<\phi<1)$ and $\gamma(\cdot)=$ $\alpha_{1}^{-1}\left(\alpha_{2}\left(\alpha_{3}^{-1}(\varpi(\cdot))\right)\right)$, respectively, where $\alpha_{1}^{-1}(\cdot)$ expresses the inverse function of the monotone function $\alpha_{1}(\cdot)$ and so does $\alpha_{3}^{-1}(\cdot)$.

Now, we rewrite the decoder-based control protocol (8) with the following compact form:

$$
u(k)=\bar{K}_{c}(\hat{x}(k)-\bar{s}(k))
$$

where $\bar{K}_{c} \triangleq I_{N} \otimes K_{c}$ and $\bar{s}(k) \triangleq \mathbb{1}_{N} \otimes s(k)$.

Next, let $w_{i}(k) \triangleq \hat{x}_{i}(k)-x_{i}(k)$ be the decoding error vector for the $i$ th node and set $w(k) \triangleq \operatorname{vec}_{N}\left\{w_{i}(k)\right\}$. In light of (56) and noting $\hat{x}(k)=w(k)+x(k)$, the closed-loop system (12) can be rewritten as

$$
e_{s}(k+1)=\left(\bar{K}_{c}+(W \otimes \Gamma)\right) e_{s}(k)+F\left(e_{s}(k)\right)+\bar{K}_{c} w(k) .
$$

Definition 3 and Lemma 4 reveal the relationship between the system state evolution and the bound of inputs. On the other hand, based on the obtained detectability analysis results, we have that the decoding error $w(k)$ is bounded. In this sense, $w(k)$ can be viewed as a bounded input of (57) and therefore the introduced ISS theory can be utilized to investigate the coding-decoding-based synchronization control problem.

Theorem 2: Under the condition in Theorem 1, the dynamical network (10) can be synchronized by the decoderbased controller (56) if there exist a positive definite matrix $R=I_{N} \otimes R_{0}>0$, a matrix $Y=I_{N} \otimes Y_{0}$ and a positive scalar $\varepsilon_{3}>0$ satisfying

$$
\Pi_{3}=\left[\begin{array}{cccc}
-R-\varepsilon_{3} \Phi_{1 \Lambda} & -\varepsilon_{3} \Phi_{2 \Lambda}^{T} & 0 & \bar{R}^{T} \\
* & -\varepsilon_{3} I_{N n} & 0 & R \\
* & * & -R & Y^{T} \\
* & * & * & -R
\end{array}\right]<0
$$

where $\bar{R}=(R(W \otimes \Gamma)+Y)$. Moreover, the desired controller gain matrix is given as

$$
K_{c}=R_{0}^{-1} Y_{0}
$$

Proof: Choosing the ISS-Lyapunov function as $V(k)=$ $e_{s}^{T}(k) R e_{s}(k)$ and calculating its difference along the trajectory of (57), one obtains

$$
\begin{aligned}
& V(k+1)-V(k) \\
\leq & {\left[\left(\bar{K}_{c}+(W \otimes \Gamma)\right) e_{s}(k)+F\left(e_{s}(k)\right)+\bar{K}_{c} w(k)\right]^{T} R } \\
& \times\left[\left(\bar{K}_{c}+(W \otimes \Gamma)\right) e_{s}(k)+F\left(e_{s}(k)\right)+\bar{K}_{c} w(k)\right] \\
& -e_{s}^{T}(k) R e_{s}(k)-\varepsilon_{3}\left[\begin{array}{c}
e_{s}(k) \\
F\left(e_{s}(k)\right)
\end{array}\right]^{T}\left[\begin{array}{cc}
\Phi_{1 \Lambda} & \Phi_{2 \Lambda}^{T} \\
* & I_{N n}
\end{array}\right] \\
& \times\left[\begin{array}{c}
e_{s}(k) \\
F\left(e_{s}(k)\right)
\end{array}\right] \\
= & \xi_{3}^{T}(k) \bar{\Pi}_{3} \xi_{3}(k)+w^{T}(k) R w(k)
\end{aligned}
$$


where

$$
\begin{aligned}
\xi_{3}(k) & =\left[\begin{array}{lll}
e_{s}^{T}(k) & F^{T}\left(e_{s}(k)\right) & w^{T}(k)
\end{array}\right]^{T}, \\
\bar{\Pi}_{3} & =\left[\begin{array}{ccc}
\bar{\Pi}_{31} & \mathbb{K}^{T} R-\varepsilon_{3} \Phi_{3 \Lambda}^{T} & \mathbb{K}^{T} \bar{K}_{c} \\
* & R-\varepsilon_{3} I_{N n} & \bar{K}_{c} \\
* & * & \bar{K}_{c}^{T} R \bar{K}_{c}-R
\end{array}\right]
\end{aligned}
$$

with $\bar{\Pi}_{31}=\mathbb{K}^{T} R \mathbb{K}-\varepsilon_{3} \Phi_{3 \Lambda}$ and $\mathbb{K}=\bar{K}_{c}+W \otimes \Gamma$.

By the Schur Complement and (59), inequality (58) means $\bar{\Pi}_{3}<0$, which yields that $V(k+1)-$ $V(k) \leq-\lambda_{\min }\left\{-\bar{\Pi}_{3}\right\}\left\|e_{s}(k)\right\|_{2}^{2}+\lambda_{\max }\{R\}\|w(k)\|_{2}^{2}$. Choosing $\alpha_{1}\left(\left\|e_{s}(k)\right\|_{2}\right)=\lambda_{\min }\{R\}\left\|e_{s}(k)\right\|_{2}^{2}, \alpha_{2}\left(\left\|e_{s}(k)\right\|_{2}\right)=$ $\lambda_{\max }\{R\}\left\|e_{s}(k)\right\|_{2}^{2}, \alpha_{3}\left(\left\|e_{s}(k)\right\|_{2}\right)=\lambda_{\min }\left\{-\bar{\Pi}_{3}\right\}\left\|e_{s}(k)\right\|_{2}^{2}$ and $\varpi\left(\|w(k)\|_{2}\right)=\lambda_{\max }\{R\}\|w(k)\|_{2}^{2}$, it is inferred from Lemma 4 that the synchronization error system (57) is input-to-state stable. So, let us choose $\beta\left(\left\|e_{s 0}\right\|_{2}, k\right)=\sqrt{\frac{\lambda_{\max }\{R\}}{\lambda_{\min }\{R\}} \phi^{k}}\left\|e_{s 0}\right\|_{2}$ and $\gamma\left(\|w(k)\|_{2}\right)=\sqrt{\frac{\lambda_{\max }^{2}\{R\}}{c \lambda_{\min }\{R\} \lambda_{\min }\left\{-\bar{\Pi}_{3}\right\}}}\|w(k)\|_{2}$, where $0<$ $c<1$. Then we have from Definition 3 that

$$
\begin{aligned}
\left\|e_{s}(k)\right\|_{2} \leq & \sqrt{\frac{\lambda_{\max }\{R\}}{\lambda_{\min }\{R\}} \phi^{k}}\left\|e_{s 0}\right\|_{2} \\
& +\sqrt{\frac{\lambda_{\max }^{2}\{R\}}{c \lambda_{\min }\{R\} \lambda_{\min }\left\{-\bar{\Pi}_{3}\right\}}}\|w(k)\|_{2} .
\end{aligned}
$$

Taking the mathematical expectation of both sides of (61), it can be immediately found from the detectability analysis result (51) and the property of "converging-input" to "converging state" discussed in Remark 3 that $\lim _{k \rightarrow \infty} \mathbb{E}\left\{\left\|e_{s}(k)\right\|_{2}\right\}=0$, which completes the proof.

\section{NUMERICAL EXAMPLES}

In this section, two simulation examples are presented to demonstrate the detectability and the effectiveness of the proposed decoder-based synchronization control protocol for the dynamical network (1).

Consider a dynamical network (1) with three nodes which are connected according to the following coupling configuration matrix

$$
W=\left[\begin{array}{ccc}
-0.2 & 0.1 & 0.1 \\
0.1 & -0.2 & 0.1 \\
0.1 & 0.1 & -0.2
\end{array}\right]
$$

and the inner-coupling matrix $\Gamma=\operatorname{diag}\{0.1,0.1\}$.

The nonlinear functions are assumed to be

$f\left(x_{i}(k)\right)=\left[\begin{array}{c}-0.5 x_{i 1}(k)+\tanh \left(0.65 x_{i 1}(k)\right)-0.15 x_{i 2}(k) \\ 1.1 x_{i 2}(k)-\tanh \left(0.95 x_{i 2}(k)\right)\end{array}\right]$.

Then, it is easy to see that the nonlinear function $f(\cdot)$ satisfies the sector bounded condition (2) with

$$
U_{1}=\left[\begin{array}{cc}
-0.5 & -0.15 \\
0 & 1.1
\end{array}\right], \quad U_{2}=\left[\begin{array}{cc}
0.15 & -0.15 \\
0 & 0.25
\end{array}\right] .
$$

The available measurements of the dynamical network described above are modeled in (1) with the following parameters $C_{1}=\left[\begin{array}{ll}0.41 & 0.52\end{array}\right], C_{2}=\left[\begin{array}{ll}0.33 & 0.44\end{array}\right], C_{3}=\left[\begin{array}{ll}0.32 & 0.60\end{array}\right]$.
In the following, we shall deal with the detectability as well as synchronization control problems for the dynamical network (1) with given parameters.

Example 1: In this example, according to our established criterion, we shall test the detectability problem for the dynamical network (1). In this case, without loss of generality, it is assumed that $u(k) \equiv 0$. Choosing $\mu_{1}=0.3$ and solving the matrix inequality (15) by the Matlab software, we have that (16) holds with $c_{0}=1.2701$. For the given scalar $\mu_{2}=0.1$ and the coding period $h=3, c_{1}=0.9435$ is obtained such that (21) holds. Moreover, the estimator gain matrices $L_{i}$ are obtained as $L_{1}=\left[\begin{array}{ll}-0.3476 & 0.8462\end{array}\right]^{T}$, $L_{2}=\left[\begin{array}{lll}-0.4161 & 1.0285\end{array}\right]^{T}$ and $L_{3}=\left[\begin{array}{lll}-0.3223 & 0.8768\end{array}\right]^{T}$. According to Theorem 1 , the packet probability $\bar{\alpha}_{i}(i=1,2,3)$ is taken as 0.1 for $q=20$.

To validate our analysis results and make our simulation non-trivial, we consider the above dynamical network which is set to be unstable. The simulation results are shown in Figs. 13 , where Figs. 1-2 plot the actual states and their decoded values for all the nodes of the network (1) and Fig. 3 shows the corresponding decoding errors $w_{i j}(k)(i=1,2,3 ; j=1,2)$. It is noticed from Fig. 3 that the errors between the actual states of the whole network and their decoded states asymptotically approach zero. Therefore, the desired detectability performance of the addressed dynamical networks is well attained.

Remark 4: For comparison, if we use the infinity-vector norm-based uniform quantization approach that has been widely used in the existing literature, we can calculate that $c_{0}=3.1110$ and $c_{1}=2.3111$ for this example, which violates the condition $0<c_{1}<1$ and the feasibility of the coding-decoding procedure cannot be guaranteed. Such a comparison result embodies the superiority of our proposed modified uniform quantization approach.

Example 2: In the second example, we consider the decoderbased synchronization control problem for the dynamical network (1). The control input signal is taken as the form in (8) for each node. By solving (58) in Theorem 2, we obtain the desired controller parameter as

$$
K_{c}=R_{0}^{-1} Y_{0}=\left[\begin{array}{cc}
0.1935 & 0.1521 \\
-0.0003 & -0.6601
\end{array}\right] .
$$

As stated in Theorem 2, the considered dynamical network can be synchronized with the designed controller parameters given above. Simulation results are presented in Figs. 4-5. From Fig. 4, it can be observed that the state response curves of all nodes diverge from the isolate node drastically when there is no control signal to the dynamical network. Fig. 5 shows that all the state response curves of the controlled network can synchronize to the isolate node according to the proposed control protocol. Therefore, the simulation examples have confirmed our theoretical analysis very well.

\section{CONCLUSions}

This paper has dealt with the synchronization control problem for a class of dynamical networks in a coding-decoding framework. By employing a modified uniform quantization 

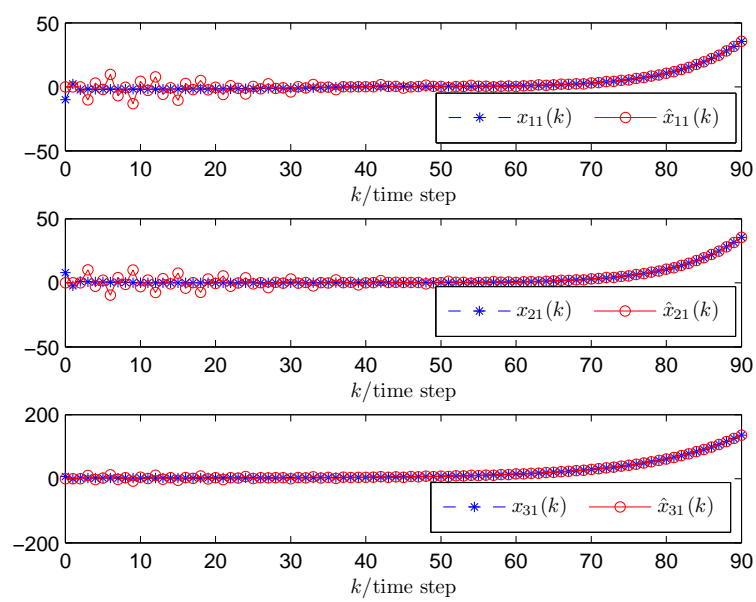

Fig. 1. State trajectories $x_{i 1}(k)$ and their decoded values $\hat{x}_{i 1}(k)(i=$ $1,2,3)$.
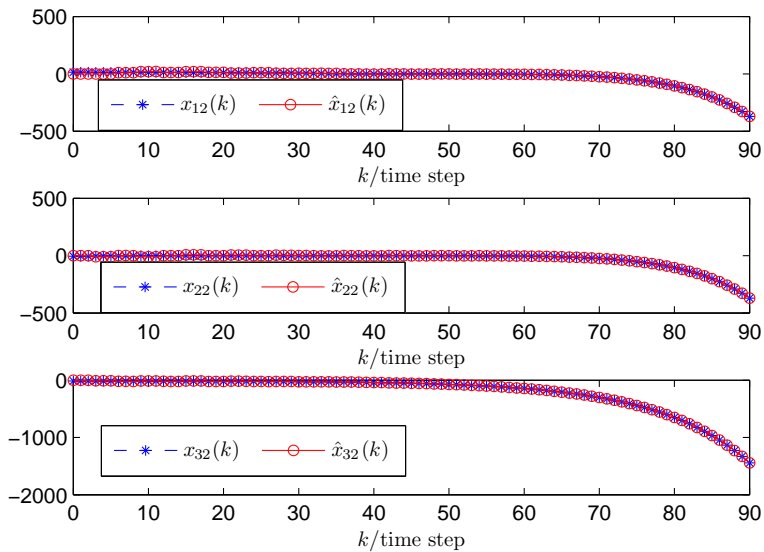

Fig. 2. State trajectories $x_{i 2}(k)$ and their decoded values $\hat{x}_{i 2}(k)(i=$ $1,2,3)$.

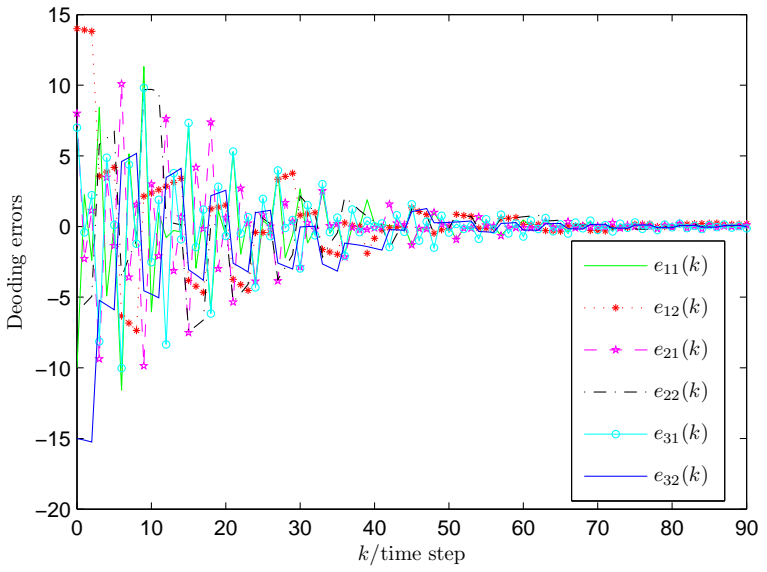

Fig. 3. Decoding errors $w_{i j}(k)(i=1,2,3 ; j=1,2)$.

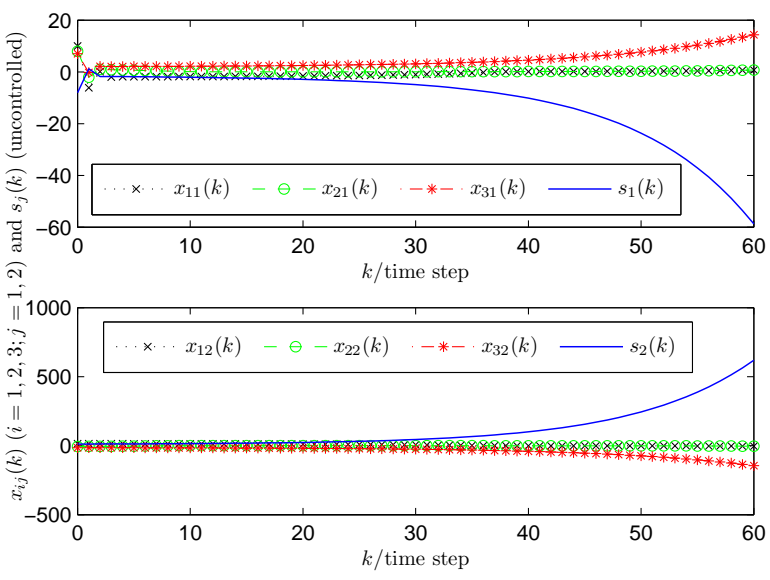

Fig. 4. State trajectories $x_{i j}(k)$ of the uncontrolled nodes $i(i=1,2,3 ; j=$ $1,2)$.

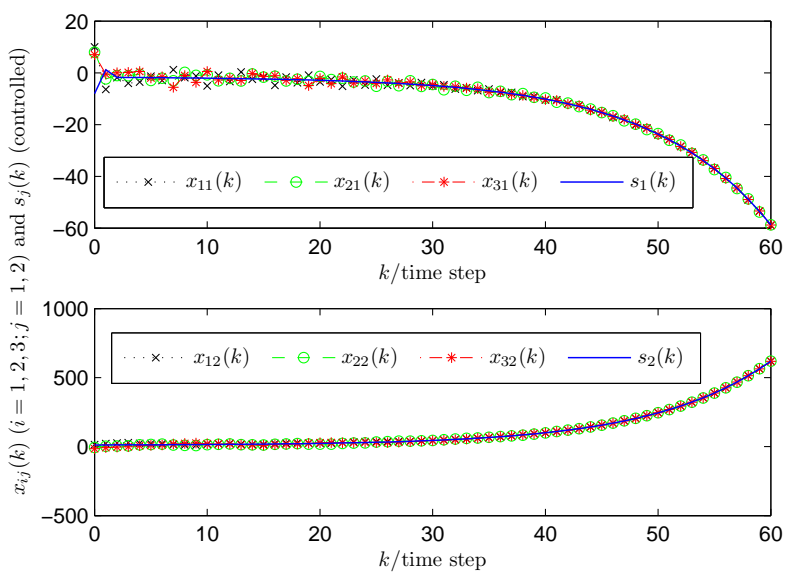

Fig. 5. State trajectories $x_{i j}(k)$ of the controlled nodes $i(i=1,2,3 ; j=$ $1,2)$.

approach, a set of coder-decoder-controller pairs has been designed to comply with the digital communication fashion. With the help of ISS theory, sufficient conditions have been derived for the closed-loop network to achieve the desired synchronization performance and the explicit expression of the controller gain matrix has been characterized in terms of the solution to certain LMIs. Finally, two illustrative examples have highlighted the detectability of the addressed dynamical network and the effectiveness of the synchronization control protocol presented in this paper. One of the future research topics would be the investigation on the coding-decoding-based state estimation problems for dynamical networks including genetic regulatory networks, sensor networks and neural networks, see e.g. [8], [9], [19], [25], [29], [36], [47], [50]. In addition, since the communication delays are inevitable during the codeword transmission via the channels [28], [32], [33], [45], [46], extending the obtained synchronization criteria to the time-delay case would be another further research topic, which is non-trivial because the delayed codewords would 
pose great complexities on the analysis of the evolution of the decoded state.

\section{REFERENCES}

[1] X. An, L. Zhang, Y. Li and J. Zhang, Synchronization analysis of complex networks with multi-weights and its application in public traffic network, Physica A-Statistical Mechanics and Its Applications, vol. 412, pp. 149-156, Oct. 2014.

[2] R. Albert, H. Jeong and A. L. Barabasi, Error and attack tolerance of complex networks, Nature, vol. 406, no. 6794, pp. 378-382, Jul. 2000.

[3] A. Berger, M. Pichler J. Klinglmayr, A. Potsch, and A. Springer Low-complex synchronization algorithms for embedded wireless sensor networks, IEEE Transactions on Instrumentation and Measurement, vol. 64, no. 4, pp. 1032-1042, Apr. 2015.

[4] H. Chen, J. Liang and J. Lu, Partial synchronization of interconnected Boolean networks, IEEE Transactions on Cybernetics, vol. 47, no. 1, pp. 258-266, Jan. 2017.

[5] H. Chen, J. Liang and Z. Wang, Pinning controllability of autonomous Boolean control networks, Science China Information Sciences, vol. 59, No. 7, Art. No. 070107, DOI: 10.1007/s11432-016-5579-8, Jul. 2016.

[6] W. Chen, Z. Jiang, X. Lu and S. Luo, $H_{\infty}$ synchronization for complex dynamical networks with coupling delays using distributed impulsive control, Nonlinear Analysis-Hybrid Systems, vol. 17, pp. 111-127, Aug. 2015.

[7] L. D. Costa, F. A. Rodrigues, G. Travieso and P. R. V. Boas, Characterization of complex networks: A survey of measurements, Advances in Physics, vol. 56, no. 1, pp. 167-242, 2007.

[8] D. Ding, Z. Wang, D. W. C. Ho and G. Wei, Distributed recursive filtering for stochastic systems under uniform quantizations and deception attacks through sensor networks, Automatica, vol. 78, pp. 231-240, Apr. 2017.

[9] D. Ding, Z. Wang, B. Shen, H. Dong and H. Shu, Distributed $H_{\infty}$ estimation with stochastic parameters and nonlinearities through sensor networks: The finite-horizon case, Automatica, vol. 48, no. 8, pp. 15751585, Aug. 2012.

[10] F. Dorfler, M. Chertkov and F. Bullo, Synchronization in complex oscillator networks and smart grids, Proceedings of the National Academy of Sciences of the United States of America, vol. 110, no. 8, pp. 2005-2010, Feb. 2013

[11] A. Farhadi and C. D. Charalambous, Robust coding for a class of sources: Applications in control and reliable communication over limited capacity channels, Systems \& Control Letters, vol. 57, no. 12, pp. 10051012, Dec. 2008.

[12] A. Farhadi and C. D. Charalambous, Stability and reliable data reconstruction of uncertain dynamic systems over finite capacity channels, Automatica, vol. 46, no. 5, pp. 1005-1012, May. 2010.

[13] L. V. Gambuzza, A. Buscarino, L. Fortuna and M. Frasca, Memristorbased adaptive coupling for consensus and synchronization, IEEE Transactions on Circuits and Systems I-Regular Papers, vol. 62, no. 4, pp. 1175-1184, Apr. 2015.

[14] X. Ge, F. Yang, and Q.-L. Han, Distributed networked control systems: A brief overview, Information Sciences, vol. 380, pp. 117-131, Feb. 2017.

[15] X. Ge, Q.-L. Han, and F. Yang, Event-based set-membership leaderfollowing consensus of networked multi-agent systems subject to limited communication resources and unknown-but-bounded noise, IEEE Transactions on Industrial Electronics, vol. 64, no. 6, pp. 5045-5054, Jun. 2017.

[16] Z. Guan, Z. Liu, G. Feng and Y. Wang, Synchronization of complex dynamical networks with time-varying delays via impulsive distributed control, IEEE Transactions on Circuits and Systems I-Regular Papers, vol. 57, no. 8, pp. 2182-2195, Aug. 2010.

[17] W. He, F. Qian, J. Lam, G. Chen, Q.-L. Han, and J. Kurths, Quasisynchronization of heterogeneous dynamic networks via distributed impulsive control: error estimation, optimization and design, Automatica, vol. 62, pp. 249-262, Dec. 2015

[18] W. He, G. Chen, Q.-L. Han, and F. Qian, Network-based leaderfollowing consensus of nonlinear multi-agent systems via distributed impulsive control, Information Sciences, vol. 380, pp. 145-158, Feb. 2017.

[19] H. Huang, G. Feng and J. Cao, Robust state estimation for uncertain neural networks with time-varying delay, IEEE Transactions on Neural Networks, vol. 19, no. 8, pp. 1329-1339, Aug. 2008.

[20] C. Hu and H. Jiang, Pinning synchronization for directed networks with node balance via adaptive intermittent control, Nonlinear Dynamics, vol. 80 , no. $1-2$, pp. 295-307, Apr. 2015
[21] Z. Jiang and Y. Wang, Input-to-state stability for discrete-time nonlinear systems, Automatica, vol. 37, no. 6, pp. 857-869, Jun. 2001.

[22] T. Li and L. Xie, Distributed coordination of multi-agent systems with quantized-observer based encoding-decoding, IEEE Transactions on Automatic Control, vol. 57, no. 12, pp. 3023-3037, Dec. 2012.

[23] X. Li, X. Wang and G. Chen, Pinning a complex dynamical network to its equilibrium, IEEE Transactions on Circuits and Systems I-Regular Papers, vol. 51, no. 10, pp. 2074-2087, Oct. 2004.

[24] X.-J. Li and G.-H. Yang, Fuzzy approximation-based global pinning synchronization control of uncertain complex dynamical networks, IEEE Transactions on Cybernetics, vol. 47, no. 4, pp. 873-883, Apr. 2017.

[25] J. Liang and J. Lam, Robust state estimation for stochastic genetic regulatory networks, International Journal of Systems Science, vol. 41, no. 1, pp. 47-63, 2010.

[26] W. Liu, Z. Wang, and M. Ni, Controlled synchronization for chaotic systems via limited information with data packet dropout, Automatica, vol. 49, no. 8, pp. 2576-2579, Aug. 2013.

[27] X. Liu, J. Cao, W. Yu, and Q. Song, Nonsmooth finite-time synchronization of switched coupled neural networks, IEEE Transactions on Cybernetics, vol. 46, no. 10, pp. 2360-2371, Oct. 2016.

[28] D. Liu, Y. Liu, and F. E. Alsaadi, A new framework for output feedback controller design for a class of discrete-time stochastic nonlinear system with quantization and missing measurement, International Journal of General Systems, vol. 45, no. 5, pp. 517-531, 2016.

[29] Y. Liu, W. Liu, M. A. Obaid, and I. A. Abbas, Exponential stability of Markovian jumping Cohen-Grossberg neural networks with mixed mode-dependent time-delays, Neurocomputing, vol. 177, pp. 409-415, 2016.

[30] J. Lu, D. W. C. Ho, and Z. Wang, Pinning stabilization of linearly coupled stochastic neural networks via minimum number of controllers, IEEE Transactions on Neural Networks, vol. 20, no. 10, pp. 1617-1629, Oct. 2009.

[31] W. Lu, Y. Han, and T. Chen, Pinning networks of coupled dynamical systems with Markovian switching couplings and event-triggered diffusions, Journal of the Franklin Institute-Engineering and Applied Mathematics, vol. 352, no. 9, pp. 3526-3545, Sep. 2015.

[32] Y. Luo, Z. Wang, G. Wei, and F. E. Alsaadi, Robust $H_{\infty}$ filtering for a class of two-dimensional uncertain fuzzy systems with randomly occurring mixed delays, IEEE Transactions on Fuzzy Systems, vol. 25 , no. 1, pp. 70-83, Feb. 2017.

[33] Y. Luo, Z. Wang, J. Liang, G. Wei, and F. E. Alsaadi, $H_{\infty}$ control for 2-D fuzzy systems with interval time-varying delays and missing measurements, IEEE Transactions on Cybernetics, vol. 47, No. 2, pp. 365-377, Feb. 2017.

[34] A. V. Savkin and T. M. Cheng, Detectability and output feedback stabilizability of nonlinear networked control systems, IEEE Transactions on Automatic Control, vol. 52, no. 4, pp. 730-735, Apr. 2007.

[35] A. V. Savkin and I. R. Petersen, Set-valued state estimation via a limited capacity communication channel, IEEE Transactions on Automatic Control, vol. 48, no. 4, pp. 676-680, Apr. 2003.

[36] B. Shen, Z. Wang, and X. Liu, Bounded $H_{\infty}$ synchronization and state estimation for discrete time-varying stochastic complex networks over a finite horizon, IEEE Transactions on Neural Networks, vol. 22, no. 1 , pp. 145-157, Jan. 2011

[37] B. Shen, Z. Wang, and X. Liu, Sampled-data synchronization control of dynamical networks with stochastic sampling, IEEE Transactions on Automatic Control, vol. 57, no. 10, pp. 2644-2650, Oct. 2012.

[38] Y. Tang, H. Gao, and J. Kurths, Robust $H_{\infty}$ self-triggered control of networked systems under packet dropouts, IEEE Transactions on Cybernetics, vol. 46, no. 12, pp. 3294-3305, Dec. 2016.

[39] L. Wang, Z. Wang, T. Huang, and G. Wei, An event-triggered approach to state estimation for a class of complex networks with mixed time delays and nonlinearities, IEEE Transactions on Cybernetics, vol. 46, no. 11, pp. 2497-2508, Nov. 2016.

[40] X. Xiao, L. Zhou, and G. Lu, Detection of singular systems via a limited communication channel with missing measurements, Information Sciences, vol. 228, no. 7, pp. 192-202, Apr. 2013.

[41] X. Yang and J. Cao, Stochastic synchronization of coupled neural networks with intermittent control, Physics Letters A, vol. 373, no. 36, pp. 3259-3272, Aug. 2009.

[42] X. Yang and D. W. C. Ho, Synchronization of delayed memristive neural networks: robust analysis approach, IEEE Transactions on Cybernetics, vol. 46, no. 12, pp. 3377-3387, Dec. 2016

[43] S. Zhai and X. Yang, Bounded synchronisation of singularly perturbed complex network with an application to power systems, IET Control Theory \& Applications, vol. 8, no. 1, pp. 61-66, Jan. 2014. 
[44] X.-M. Zhang and Q.-L. Han, A decentralized event-triggered dissipative control scheme for systems with multiple sensors to sample the system outputs, IEEE Transactions on Cybernetics, vol. 46, no. 12, pp. 27452757, Dec. 2016.

[45] X.-M. Zhang and Q.-L. Han, Network-based $H_{\infty}$ filtering using a logic jumping-like trigger, Automatica, vol. 49, no. 9, pp. 1428-1435, May 2013.

[46] X.-M. Zhang, Q.-L. Han, and X. Yu, Survey on recent advances in networked control systems, IEEE Transactions on Industrial Informatics, vol. 12, no. 5, pp. 1740-1752, Oct. 2016.

[47] J. Zhang, L. Ma, and Y. Liu, Passivity analysis for discrete-time neural networks with mixed time-delays and randomly occurring quantization effects, Neurocomputing, vol. 216, pp. 657-665, 2016.

[48] W. Zhang, Z. Wang, Y. Liu, D. Ding, and F. E. Alsaadi, Event-based state estimation for a class of complex networks with time-varying delays: a comparison principle approach, Physics Letters A, vol. 381, no. 1, pp. 10-18, 2017.

[49] L. Zhou and G. Lu, Detection and stabilization for discrete-time descriptor systems via a limited capacity communication channel, Automatica, vol. 45, no. 10, pp. 2272-2277, Oct. 2009.

[50] L. Zou, Z. Wang, H. Gao, and X. Liu, Event-triggered state estimation for complex networks with mixed time delays via sampled data information: the continuous-time case, IEEE Transactions on Cybernetics, vol. 45, no. 12, pp. 2804-2815, Dec. 2015.

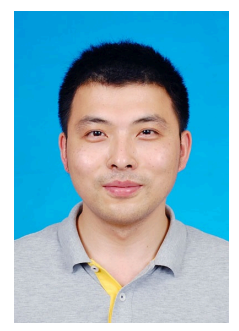

Licheng Wang received the B.Sc. degree in automation in 2011 from Weifang University, Weifang, China, and the M.Sc. degree in control science and engineering in 2014 from University of Shanghai for Science and Technology, Shanghai, China. He is now currently pursuing the Ph.D. degree in Control Science and Engineering at the University of Shanghai for Science and Technology, Shanghai, China. Since Nov. 2016, he has been a visiting $\mathrm{Ph} . \mathrm{D}$. student in the Department of Electronic and Computer Engineering at Brunel University London in the UK. His research interests include nonlinear stochastic control and filtering, as well as complex networks and sensor networks.

Mr. Wang is a reviewer for some international journals.

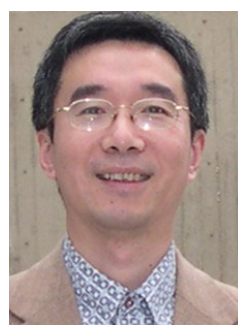

Zidong Wang (SM'03-F'14) was born in Jiangsu, China, in 1966. He received the B.Sc. degree in mathematics from Suzhou University, Suzhou, China, in 1986, and the M.Sc. degree in applied mathematics and the Ph.D. degree in electrical engineering from the Nanjing University of Science and Technology, Nanjing, China, in 1990 and 1994, respectively.

He is currently a Professor of dynamical systems and computing with the Department of Information Systems and Computing, Brunel University London, Uxbridge, U.K. From 1990 to 2002, he held teaching and research appointments in universities in China, Germany, and the U.K. He has published over 300 papers in refereed international journals. His current research interests include dynamical systems, signal processing, bioinformatics, control theory, and applications.

Prof. Wang was a recipient of the Alexander von Humboldt Research Fellowship of Germany, the JSPS Research Fellowship of Japan, and the William Mong Visiting Research Fellowship of Hong Kong. He serves (or has served) as the Editor-in-Chief of Neurocomputing, and an Associate Editor for 12 international journals, including the IEEE TRANSACTIONS ON AUTOMATIC CONTROL, the IEEE TRANSACTIONS ON CONTROL SYSTEMS TECHNOLOGY, the IEEE TRANSACTIONS ON NEURAL NETWORKS, the IEEE TRANSACTIONS ON SIGNAL PROCESSING, and the IEEE TRANSACTIONS ON SYSTEMS, MAN, AND CYBERNETICSPART C: APPLICATIONS AND REVIEWS. He is a Fellow of the Royal Statistical Society and a member of program committee for several international conferences.

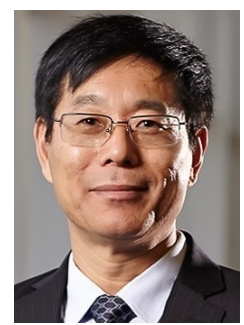

Qing-Long Han (M'09-SM'13) received the B.Sc. degree in mathematics from Shandong Normal University, Jinan, China, in 1983, and the M.Sc. and $\mathrm{Ph} . \mathrm{D}$. degrees in Control Engineering and Electrical Engineering from East China University of Science and Technology, Shanghai, China, in 1992 and 1997, respectively.

From 1997 to 1998 , he was a Post-doctoral Researcher Fellow with the Laboratoire d'Automatique et d'Informatique Industielle (now renamed as Laboratoire d'Informatique et d'Automatique pour les Systémes), Ecole Sup érieure d'Ing'enieurs de Poitiers (now renamed as Ecole Nationale Supérieure d'Ingé nieurs de Poitiers), Universit é de Poitiers, France. From 1999 to 2001, he was a Research Assistant Professor with the Department of Mechanical and Industrial Engineering, Southern Illinois University Edwardsville, Edwardsville, IL, USA. From 2001 to 2014, he was a Laureate Professor, the Associate Dean of Research and Innovation with the Higher Education Division, and Founding Director of the Centre for Intelligent and Networked Systems, Central Queensland University, Rockhampton, QLD, Australia. From 2014 to 2016, he was the Deputy Dean of Research with the Griffith Sciences, and a Professor with the Griffith School of Engineering, Griffith University, Nathan, QLD, Australia. In 2016, he joined Swinburne University of Technology, Melbourne, VIC, Australia, where he is currently the Pro Vice-Chancellor of Research Quality and a Distinguished Professor. In 2010, he was appointed as a Chang Jiang (Yangtze River) Scholar Chair Professor by the Ministry of Education, Beijing, China. His current research interests include networked control systems, neural networks, time-delay systems, multi-agent systems, and complex dynamical systems.

Prof. Han was a recipient of one of the World's Most Influential Scientific Minds from 2014 to 2016, and the Highly Cited Researcher Award in Engineering by Thomson Reuters. He is an Associate Editor of a number of international journals, including the IEEE TRANSACTIONS ON INDUSTRIAL ELECTRONICS, the IEEE TRANSACTIONS ON INDUSTRIAL INFORMATICS, the IEEE TRANSACTIONS ON CYBERNETICS, and the Information Sciences.

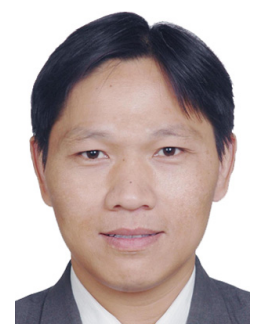

Guoliang Wei received the B.Sc. degree in mathematics from Henan Normal University, Xinxiang, China, in 1997 and the M.Sc. degree in applied mathematics and the Ph.D. degree in control engineering, both from Donghua University, Shanghai, China, in 2005 and 2008, respectively. He is currently a Professor with the Department of Control Science and Engineering, University of Shanghai for Science and Technology, Shanghai, China.

From March 2010 to May 2011, he was an Alexander von Humboldt Research Fellow in the Institute for Automatic Control and Complex Systems, University of DuisburgEssen, Germany. From March 2009 to February 2010, he was a post doctoral research fellow in the Department of Information Systems and Computing, Brunel University, Uxbridge, UK, sponsored by the Leverhulme Trust of the UK From June to August 2007, he was a Research Assistant at the University of HongKong. From March to May 2008, he was a Research Assistant at the City University of Hong Kong.

His research interests include nonlinear systems, stochastic systems, and bioinformatics. He has published more than 20 papers in refereed international journals. He is a very active reviewer for many international journals. 